\title{
Sexual mixing and the risk environment of sexually active transgender women: data from a respondent-driven sampling study of HIV risk among transwomen in San Francisco, 2010
}

\author{
Erin C Wilson", Glen-Milo Santos and H Fisher Raymond
}

\begin{abstract}
Background: Research on the sexual networks of transwomen is central to explaining higher HIV risk for this population. This study examined HIV risk behaviors and sexual mixing patterns of transwomen by demographic and HIV-related risk behaviors.

Methods: Data were obtained from a 2010 study of HIV risk for transwomen in San Francisco. Assortativity by race, partner type, HIV serostatus, and IDU across sexual networks was calculated using Newman's assortativity coefficient (NC). Multivariable generalized estimating equations (GEE) logistic regression models were used to evaluate associations between unprotected anal intercourse with race and HIV serostatus, partner-IDU status and relationship type discordance while adjusting for the HIV status of transwomen.
\end{abstract}

Results: There were 235 sexually active transwomen in this study, of whom 104 (44.3\%) were HIV-positive and 73 (31.1\%) had a history of injection drug use. Within the 575 partnerships, African American/black and Latina transwomen were the most racially assortative ( $\mathrm{NC} 0.40,95 \% \mathrm{Cl} 0.34-0.45$, and $\mathrm{NC} 0.43,95 \% \mathrm{Cl} 0.38-0.49$, respectively). In partnerships where the partner's HIV status was known $(n=309,53.7 \%)$, most transwomen were in sexual partnerships with people of their same known serostatus $(71.8 \%, n=222)$. In multivariable analyses, unprotected anal intercourse was significantly associated with primary partners, having a sexual partner who was an injection drug user, and sexual partner seroconcordance.

Conclusions: Public health efforts to reduce transwomen's HIV risk would likely benefit from prioritizing prevention efforts to risk reduction within IDU-discordant and primary partnerships, determining risks attributable to sexual network characteristics, and actively addressing injection drug use among transwomen.

\section{Background}

A systematic review of HIV research in the US found that transgender women (transwomen) engage in high rates of unprotected receptive anal intercourse, which may explain elevated rates of HIV [1]. Findings from epidemiological research on HIV suggests that members engaging in serodiscordant partnering, or sex between partners with different HIV statuses, is key to maintaining and transmitting HIV within populations at risk and their sexual partners [2]. Past studies of transwomen have found that sexual risk behavior is highest in primary

\footnotetext{
* Correspondence: erin.wilson@sfdph.org

San Francisco Department of Public Health, San Francisco, California
}

sexual partnerships [3-5]. However, one recent study found that risk for HIV was highest for transwomen who did sex work, possibly due to the higher volume of sexual partnerships by choice or by assault that increased chances for exposure to HIV [6]. Transwomen have also been found to be more likely than other populations at risk for HIV to have serodiscordant partnerships, though partner serodiscordance did not significantly influence engagement in unprotected receptive anal intercourse [7]. HIV risk may, in part, be explained by the behaviors specific to particular partner types and the serostatus of those partners.

Transwomen may also be at risk for HIV due to substance use. A number of studies have demonstrated 
elevated use of illicit drugs among transwomen $[1,8]$. A recent population-based study of transwomen found that testing positive for HIV was significantly related to methamphetamine use, methamphetamine use before or during anal intercourse, and at least weekly methamphetamine use [9]. Concerns about HIV transmission via injection drug use among transwomen have been related to needle sharing practices, injection of hormones or other fillers (i.e. illicit "silicone") and injection drug use behaviors of sexual partners [10-12]. Risk related to injection hormones is mixed as one study found that a combination measure of injection drug and hormone use to be related to self-reported HIV infection, while another recent study of injection of fillers (i.e. illicit substances used to feminize transwomen's appearance) found no association with an HIV positive test $[8,13]$. A population-based study of transwomen in San Francisco found that $32.5 \%$ had ever injected substances not prescribed by medical professionals and $11.8 \%$ had injected in the past 12 months [14]. In the same study, injection drug use in the past 12 months was one of only three factors that remained significantly associated with HIV infection. Transwomen who inject drugs may be at high risk for HIV from non-sterile injection equipment, and from sexual transmission, as this mode of transmission accounts for a substantial number of incident infections in some studies $[15,16]$. However, data are missing on HIV risk related to sex with injection drug users among transwomen, thus it is difficult to discern whether IDUrelated risk among transwomen is due to injection drug use practices of transwomen, their sexual partners who are IDU, or both.

By comparison, race has been an important sexual mixing factor as it relates to HIV risk among MSM [17]. Bohl et. al's [17] article on racial disparities in HIV found that the sexual networks of African American/black MSM account for their higher prevalence of HIV compared with non-African American/black MSM. This study provided compelling evidence that sexual networks rather than individual characteristics or behaviors explained significantly higher risk of HIV for African American/black MSM [17]. The systematic review of HIV among U.S. transwomen found that the weighted mean HIV prevalence for African American/blacks was 56.3\%, which was almost twice as high as that found among White or Hispanic transwomen [1]. An important next step in the investigation of HIV risk for transwomen is to better understand risk via their sexual networks. To fill a void in what we know about the sexual partnerships of transwomen, we conducted an analysis of demographic and risk factors of the sexual networks of sexually active transwomen in San Francisco. We also evaluated which sexual mixing factors by race, partner type, IDU status, and HIV status were correlated with HIV-related sexual risk behaviors.

\section{Methods}

\section{Respondent-level variables - transwomen participants}

Data for this analysis are taken from a study of transgender women that took place in 2010 (TEACH- the Transgender Empowerment and Community Health study). Transgender women were recruited using respondent driven sampling (RDS) in which 11 initial seed participants recruited a total final sample of 314 transwomen [18]. Individuals were eligible for the study if they (1) selfidentified as transwomen, (2) were age 18 years or older and (3) reported living in San Francisco. Each study participant was screened for study eligibility prior to enrollment. Eleven seeds diverse in respect to race/ethnicity, income, education, and age were selected as the initial recruits. Recruits could be anyone in their social network who seeds (or recruiters) thought was eligible for the study. All participants were remunerated $\$ 10$ for recruiting peers who were eligible and enrolled into the study (see Rapues et al., 2013 for more information on seeds). All data on HIV status were collected by either self-report and/or through an HIV assay. Respondents who were HIV-positive were able to refuse the HIV test with no repercussions for receiving an incentive.

Participants were asked to report on up to 5 sexual partners from the past six months, including each partner's age, gender identity, race/ethnicity, what type of partner each person was (i.e. primary, casual or commercial), where the participant met each partner, sexual behavior, HIV status and whether that person was an injection drug user. We created a dataset of all the sexual partnerships of TEACH participants for the past six months. Those that did not report having a sexual partner in the last six months, those with missing data on HIV-serostatus, and those who were HIV-positive but with an unknown positive status at the time of participation were excluded from this analysis.

\section{Statistical analysis}

We analyzed sexual partner mixing by race, HIV serostatus, and explored partner-level characteristics including relationship type and IDU status. Consistent with surveillance and other sexual mixing studies, partner data analyzed for this study are egocentric and therefore based solely on data reported by transwomen participants, not from their partners [19]. Sexual risk in TEACH was assessed by asking a number of questions about characteristics and behaviors of the last 5 sexual partners of transwomen and by asking transwomen what sexual behaviors they engaged in with each of these partners. This strategy is employed nationally in the U.S. and in global HIV surveillance efforts to enhance recall and obtain specificity in recent sexual behaviors of populations most at risk for HIV [20]. 
Transwomen were also asked to identify the race, HIV status, and injection drug use status of their last 5 sexual partners. Partner types were categorized as main, casual and commercial. Main partners were defined as, "someone who is your primary sexual partner and you feel committed to (boyfriend, lover, husband, girlfriend, wife)". Casual partners were defined as, "someone you have sex with, but don't feel committed to or don't know very well". Commercial partners were defined as, "someone you had sex with in exchange for things like money or goods".

Sexual mixing in HIV status or serodiscordance was defined as a difference in reported or tested HIV status of the transwomen participant and the reported HIV status of the sexual partner, as has been done in studies of MSM [21,22]. We excluded 47 partnerships where the HIV status of the transwoman participant was unknown. In the case that a transwoman participant was HIV positive and reported her partner's HIV status as negative, and vice versa for a transwoman who was HIV-negative and reported her partner as being HIV-positive, the partnership was categorized as serodiscordant. Transwomen's partnerships with those of an unknown HIV status were also categorized as serodiscordant, consistent with prior literature $[23,24]$. We calculated assortativity by race, partner status (main, casual, or commerical), HIV serostatus, and IDU between transwomen and their sexual networks using Newman's assortativity coefficient. Newman's assortativity coefficients ranges from -1 to 1 ; negative coefficients correspond with disassortativity or a higher-degree of mixing, while positive coefficients correspond with assortativity or a lower-degree of mixing [25]. A study of MSM in San Francisco over three waves of data collection from 20042011 found slightly assorative mixing by race overall at $r=0.08$ in 2004, but highly assortative partnering for African Americans/blacks $(r=0.44)$ [26]. Based on this study and the work of Doherty et al. [27], we have categorized mixing coefficient values $\geq 0.35$ as assortative, $0.15-$ 0.34 as moderately assortative, and $<0.15$ as disassortative.

\section{Multivariable analysis of engagement in sexual risk behavior}

In the primary outcome multivariable analysis, we evaluated the associations between UAI with sexual partners and discordance in race and HIV serostatus, as well as partner-IDU status and relationship type, while adjusting for the HIV status of transwomen. We used multivariable generalized estimating equations (GEE) logistic regression models that accounted for correlation between the multiple partnerships of each study participant to estimate odds ratios and 95\% confidence intervals.

Analyses were conducted using STATA 12.0. Data adjustments to make inference to the population from which the sample was drawn were not made because the sample was stratified for this analysis and the exclusion of 79 participants eliminates the ability to assure equilibrium in the sample. Therefore, findings from this study describe findings from this dataset alone and may not be generalizable to the San Francisco transwoman population overall.

This study received human participants review and approval from the University of California, San Francisco's Committee on Human Research.

\section{Results}

\section{Participants demographics}

Of the 314 TEACH participants, 15 refused to take an HIV test and 64 did not have any sexual partners in the past six months, thus leaving 235 transwomen for our analysis. Among these sexually active transwomen $44.3 \%$ ( $n=104$ ) were HIV-positive (Table 1 ). Of the 235 sexually active transwomen, $72.3 \%(n=170)$ reported having five or fewer sexual partners. The range of sexual partnerships per transwomen was 1-500, with a median number of three (inter-quartile range [IQR]: 1-6) sexual partners and a mean of 11 (standard deviation: 39.01). However, not all sexual partnerships were included in this analysis as participants were only asked about characteristics of up to five individual sexual partners. Within this dataset, a respondent may have had her data repeated, but each partnership was unique.

Over $60 \%$ of the sample was transwomen who were either Latina (30.6\%) or African American/black (31.1\%). Whites made up $15.3 \%$ of the sample, $16.6 \%$ were "Other" and only $6.4 \%$ of our sample was Asian or Pacific Islander (API). Transwomen split almost evenly into identifying as transgender or female $(52.3 \%$ and $47.7 \%$, respectively). The median age of transwomen in this analysis was 42 (IQR 32-48) and the mean was 41 (SD 10.7). Most transwomen did not have a lifetime history of IDU ( $\mathrm{n}=162,68.9 \%)$, but a sizeable proportion did report being IDU $(n=73,31.1 \%)$.

\section{Partners demographics}

There were a total of 575 partnerships analyzed from the 235 sexually active transwomen respondents. The characteristics of these partners are summarized in Table 1. Most partners of transwomen were between the ages of 26-43 (50\%). The mean age of partners was 34.8 (SD 15). The majority of partnerships were with men $(\mathrm{n}=537,93.4 \%)$, and the most common place where transwomen met their sexual partners was on the street $(\mathrm{n}=225,39.1 \%)$. The majority of sexual partnerships were casual ( $n=322,56 \%)$, while $26.3 \%(n=151)$ were with primary partnerships and $17.7 \%(n=102)$ were commercial relationships. 
Table 1 Characteristics of sexually active transwomen participants $(n=235)$ and their sexual partners in the last 6 months ( $n=575)$

\begin{tabular}{lll}
\hline $\begin{array}{l}\text { Transwomen participant demographics } \\
\text { and behaviors }\end{array}$ & $\mathbf{n}$ mean & (\%) (SD) \\
\hline $\begin{array}{l}\text { Age, mean (SD) } \\
\text { Gender Identity }\end{array}$ & 41 & $(10.7)$ \\
$\quad$ Female & 112 & $(47.7)$ \\
$\quad$ Transfemale & 123 & $(52.3)$ \\
Race/Ethnicity & & \\
African American/black & 73 & $(31.1)$ \\
Asian or Pacific Islander & 15 & $(6.4)$ \\
Latina/Hispanic & 72 & $(30.6)$ \\
White & 36 & $(15.3)$ \\
Other & 39 & $(16.6)$
\end{tabular}

Injection drug use

No

Yes

HIV Status

Negative

Positive

Any Unprotected Anal Intercourse with sexual partners

No

Yes

Sexual partner demographics and behavior

Age, mean (SD)

Gender Identity

Male

Female

Transwoman

Transman

Other/Did not specify

Race/Ethnicity

African American/black
Asian or Pacific Islander
Latino/Hispanic
White
Other

Relationship with participant

Primary

Casual

Commercial

Meeting site

On the street, park or public place

Introduced by friends

Internet
Table 1 Characteristics of sexually active transwomen participants $(n=235)$ and their sexual partners in the last 6 months $(n=575)$ (Continued)

\begin{tabular}{lll}
\hline Bar & 57 & $(9.9)$ \\
Other & 164 & $(28.5)$ \\
Injection drug use & & \\
No & 520 & $(90.4)$ \\
Yes & 55 & $(9.6)$ \\
HIV Status & & \\
Positive & 241 & $(41.9)$ \\
Negative & 68 & $(11.8)$ \\
Unknown & 266 & $(46.3)$ \\
Same HIV-status as participant & & \\
No & 353 & $(61.4)$ \\
Yes & 222 & $(38.6)$ \\
Same race as participant & & \\
No & 365 & $(36.5)$ \\
Yes & 210 \\
Unprotected Anal Intercourse with participant & & \\
No & & \\
Yes & $452.5)$ \\
\hline
\end{tabular}

\section{Sexual partners and mixing}

HIV

Among transwomen with a known HIV status, almost half of transwomen's sexual partnerships were with partners of an unknown HIV status $(46.3 \%, n=266)$. Of those who knew their partner's HIV status $(n=309$, $53.7 \%$ ), transwomen were in more sexual partnerships with people of their same known serostatus (36.5\%, $\mathrm{n}=222$ ). Among sexual partners of transwomen with a known HIV serostatus, the Newman's coefficient was 0.40 (0.33-0.47), suggesting that transwomen were assortative in their sexual partnering with people of the same serostatus. Excluding partnerships where the HIV status was unknown for partners $(n=266), 25 \%$ of partnerships were between HIV-positive transwomen and HIV-negative partners $(n=78,24.3 \%)$. Only $3 \%$ of partnerships were between HIV-negative transwomen and an HIV-positive sexual partner $(n=9)$.

\section{IDU}

Most transwomen did not ever inject drugs, though a sizeable portion did inject drugs $(n=73,31.1 \%)$. Similarly, most transwomen reported that their partners did not inject drugs $(n=520,90.4 \%)$, while $9.6 \%$ of partners $(n=55)$ reportedly did inject drugs. The Newman's assortativity coefficient for IDU was 0.23 (95\% CI 0.19- 0.26), suggesting that transwomen were moderately more likely to partner with people of the same IDU status. Sexual partnerships where non-IDU transwomen had partners who were IDU 
made up $13 \%$ of IDU-discordant partnerships $(n=19)$, while sexual partnerships where IDU transwomen had non-IDU partners made up the other $87 \%(\mathrm{n}=123)$. Therefore, more transwomen were IDUs in sexual partnerships where IDU status was mixed.

\section{Race}

The majority of sexual partners of transwomen were White (195 of 575 partners; 33.9\%), African American/black (183 of 575 partners; $31.8 \%$ ), or Latino (134 of $575 ; 23.3 \%$ ) (Table 1). However, when assessing partnerships on a raceby-race basis, African-American/black, Latino, and White transwomen most commonly reported having sexual partners of the same race. Sexual partners of White transwomen were $47 \%$ White $(n=43)$, followed by AfricanAmerican/black 26\% $(\mathrm{n}=24)$, and Latino 13\% $(\mathrm{n}=12)$. Sexual partners of African-American/black transwomen were $60 \%$ African-American/blacks $(\mathrm{n}=106)$, followed by White 29\% $(n=40)$ and Latino $8 \%(N=15)$. Sexual partners of Latino transwomen were $49 \%$ Latino $(n=100)$, followed by white $26 \%(\mathrm{n}=53)$ and African-American/ black $11 \%(\mathrm{n}=24)$. The overall Newman's assortativity coefficient for race was .26 (95\% CI 0.22-0.30), suggesting that transwomen were moderately assortative by race. When distilled by race, the assortativity coefficient by Asian race was 0.03 (95\% CI $0.01-0.05)$, by African American/black was 0.40 (95\% CI 0.34-0.45), by Latino was 0.43 (95\% CI $0.38-0.49)$ and by White was 0.11 (95\% CI 0.06-0.15). Thus, African American/black and Latina transwomen were more assortative than Whites and Asians, and had sexual partners of the same race more often that transwomen of other races.

\section{Prevalence and multivariate analysis of engagement in sexual risk behavior}

Among transwomen, 88 (37.5\%) reported having UAI with at least one sexual partner. Transwomen reported having UAI in 123 sexual partnerships $(21.4 \%$ of 575 partners). The results of the GEE multivariable logistic regression model examining the association of sexual mixing factors with unprotected anal sex are summarized in Table 2. Results indicate that compared to primary partners, transwomen had an adjusted odds of UAI that was $0.23(95 \% \mathrm{CI} 0.15-0.36, \mathrm{p}<0.001)$ and $0.20(95 \%$ CI $0.10-0.43, \mathrm{p}<0.001$ ) lower with casual and commercial partners, respectively. Moreover, transwomen had an adjusted odds of UAI of 0.55 (95\% CI $0.35-0.87, \mathrm{p}<0.001$ ) lower with HIV serodiscordant partners compared to seroconcordant partners. In addition, transwomen had an adjusted odds of UAI that was 2.66 (95\% CI 1.434.97, $\mathrm{p}<0.001)$ greater with sexual partners who were IDU than with non-IDU. The odds of engaging in UAI were not significantly different between same race and different race partners of transwomen (aOR 0.77, 95\% CI 0.50-1.21, $\mathrm{p}=.40$ ).

\section{Discussion}

The goal of this analysis was to determine if there were significant sexual mixing patterns in addition to individual risk behaviors to help understand high HIV risk among transwomen. In the final analysis we found that UAI was significantly associated with having a sexual partner who was an injection drug user, primary partner, and/or HIV seroconcordant. Transmission of HIV from injection drug users to sexual partners who are uninfected may be a source of HIV risk for transwomen. Sexual transmission of HIV from seropositive IDUs to non-injecting sexual partners is extremely likely, and more risky than that associated with sharing needles [25]. In this study, only $10 \%$ of partners were IDU, but that represented 55 sexual encounters for transwomen, thus mixing with individuals who are injection drug users remains an important area of investigation for understanding transwomen's risk for HIV.

Table 2 Prevalence and Multivariable GEE logistic regression model on Partner-level correlates of Unprotected Anal Intercourse

\begin{tabular}{|c|c|c|c|c|c|c|c|c|c|c|c|}
\hline & & \multicolumn{4}{|c|}{ UAI with partners } & \multicolumn{3}{|c|}{ Crude } & \multicolumn{3}{|c|}{ Adjusted* } \\
\hline & & No & (\%) & Yes & (\%) & OR & $95 \% \mathrm{Cl}$ & P-value & AOR & $95 \% \mathrm{Cl}$ & P-value \\
\hline & Same race partners & 252 & $(74.1)$ & 88 & $(25.9)$ & 1 & - & - & 1 & - & \\
\hline & Different race partners & 200 & $(85.1)$ & 35 & $(14.9)$ & 0.80 & $0.54-1.20$ & 0.28 & 0.77 & $0.50-1.21$ & 0.26 \\
\hline & Primary partner & 87 & (57.6) & 64 & $(42.4)$ & 1 & - & - & 1 & - & - \\
\hline & Casual partner & 279 & $(86.6)$ & 43 & $(13.4)$ & 0.21 & $0.13-0.32$ & $<0.001$ & 0.23 & $0.15-0.36$ & $<0.001$ \\
\hline \multirow[t]{5}{*}{ Partner Characteristics } & Commercial partner & 86 & $(84.3)$ & 16 & $(15.7)$ & 0.18 & $0.09-0.37$ & $<0.001$ & 0.20 & $0.10-0.43$ & $<0.001$ \\
\hline & Non-IDU partners & 420 & $(80.8)$ & 100 & $(19.2)$ & 1 & - & - & 1 & - & - \\
\hline & IDU partners & 32 & $(58.2)$ & 23 & $(41.8)$ & 2.44 & $1.37-4.33$ & 0.002 & 2.66 & $1.43-4.97$ & 0.002 \\
\hline & HIV seroconcordant partners & 154 & $(69.4)$ & 68 & (30.6) & 1 & - & - & 1 & - & \\
\hline & HIV serodiscordant partners & 298 & $(84.4)$ & 55 & $(15.6)$ & 0.43 & $0.29-0.64$ & $<0.001$ & 0.55 & $0.35-0.87$ & 0.01 \\
\hline
\end{tabular}

Notes: *Model adjusted for participant-level characteristics in table above and individual-level co-variables (e.g. Age and HIV-status of TEACH participant). GEE models adjusted for clustering of partner-level data by TEACH participant. 
These data also support prior studies that have shown higher rates of UAI within primary partnerships $[3,4]$. Of all three partner types - primary, casual and commercial primary partners have consistently been the type in which transwomen engage in the highest risk behavior, which is consistent with the literature for MSM, IDU and heterosexual men and women [3,4,28-32]. Yet transwomen in this analysis were also less likely to engage in UAI with serodiscordant partners. Many of the partnerships analyzed as serodiscordant were those in which transwomen did not know the HIV status of their sexual partner, which made up almost of half of sexual partnerships. The finding on partnerships of unknown HIV status is higher than recent research with Peruvian transwomen and MSM showing that one quarter of partners were people of unknown serostatus [33]. Yet findings that transwomen were significantly less likely to engage in UAI with partners of a different serostatus is consistent with other research findings [7], and may also be a sign that transwomen are serosorting. Available data on the effects of serosorting on HIV transmission are equivocal; some studies suggest protective benefits [34], while some have found increased risk for transmission due to serosorting [12]. Given the disproportionate impact of HIV among transwomen, it would be prudent to inform transwomen about possible limitations of serosorting in reducing HIV risk. Also different, most serodiscordant partnerships in our study were characterized by transwomen living with HIV. As has been found in research with HIV-positive MSM, transwomen already living with HIV many may be choosing HIV-negative partners to protect from super-infection and other sexually transmitted diseases [35]. Alternatively, this finding may represent the overall high prevalence of HIV among transwomen and the high likelihood of having a HIV-negative partner.

One risk we did not observe in this study was an association between same race sexual partnerships and risk for HIV via elevated rates of engagement in UAI. Highly interconnected networks among African American/black MSM have been a driver of HIV in the population [1,14,36]. African American/black transwomen have almost twice the rate of HIV as the overall transwomen population [18]. Assortativity was significantly higher for African American/black and Latina participants and engagement in UAI was not significantly different. Thus, findings of assortativity on race alone may mean highly interconnected sexual networks with those of their same racial/ethnic identities are important for understanding higher HIV risk among African American/black and Latina transwomen. Given the lack of significant differences in engagement in sexual risk behavior, this study provides evidence that same-race partnerships may be a driver of HIV risk for transwomen. More work needs to be done to see if transwomen are facing the same risks for HIV as African
American/black MSM that was attributed to sexual networks despite low engagement in sexual risk behaviors.

Finally, data from this analysis point to a diversity of transwomen's choices in sexual partners by race. Though the largest proportion of sexual partners were the same race as the transwomen participants, up to $70 \%$ of partners were of a different race. The only known study of the main male partners of transwomen in which a convenience sample was used to recruit participants found mostly African American/black male partners [37]. The difference in findings on the race of sexual partners between studies may be due to the population-based sampling conducted for this study that points to diverse rather than limited sexual mixing patterns among transwomen overall.

There are a number of limitations to this study. Data were gathered based on self-report, which may be inherently biased due to social desirability. Demographic and behavioral factors of sexual partners were also reported by transwomen respondents and not the partners themselves. Therefore, the characteristics and behaviors of sexual partners may be inaccurate due to biases of the participant, inaccurate knowledge and recall bias. Though these data are taken from a study employing respondent driven sampling, the exclusion of participants who did not recently have a sexual partner may make these data less generalizable to the overall transwoman population in San Francisco.

\section{Conclusions}

These data point to important next steps in research and prevention. Past prevention research has mostly targeted transwomen, and these data suggest that serving one side of the risk equation may not be enough to curb the epidemic within this hard hit population [38]. Indeed, it may be the lack of prevention resources provided to the sexual partners of transwomen that has contributed to the persistent high risk for HIV among transwomen, thus prevention messaging must be designed and targeted to sexual partners too. Research to better understand the considerations of transwomen when selecting sexual partners, such as the convergence with IDU sexual networks, may contribute to more targeted HIV and IDU prevention and harm reduction strategies that impact transwomen's own drug use behavior and selection of partners who are IDUs [11]. Interventions that work with transwomen to identify ways to ask about the serostatus of their sexual partners are also warranted. Serosorting is only protective when two individuals can make safe sex decisions based on known HIV status. The promise of home testing and instant HIV testing may help further such efforts and should be considered for distribution among transwomen and for their sexual partners. Finally, future longitudinal research with transwomen is also needed so analyses can better 
determine risks among incident cases instead of extrapolating findings from cross sectional data within high prevalence samples.

\section{Competing interests}

The authors declare that they have no competing interests.

\section{Authors' contributions}

HFR designed, implemented, and supervised the conduct of this study. ECW conceived of the analysis, and drafted the manuscript. HFR, ECW and GMS contributed to the study design for this analysis and GMS conducted the statistical analyses. HFR, GMS, and ECW contributed to the writing and editing of initial drafts and approved the final draft of the article.

\section{Acknowledgments}

We thank the participants for their time and investment into the gathering of these data. We also acknowledge the staff of the San Francisco Department of Public Health who took their valuable time out to make this study happen, including the HIV Prevention Section and HIV Epidemiology Section for their time, presence and skills in conducting this study.

Received: 10 April 2014 Accepted: 23 July 2014

Published: 6 August 2014

\section{References}

1. Herbst JH, Jacobs ED, Finlayson TJ, Jacobs ED, Finlayson TJ, McKleroy VS Neumann MS, Crepaz N: Estimating HIV prevalence and risk behaviors of transgender persons in the United States: a systematic review. AIDS Behav 2008, 12(1):1-17.

2. Friedman SR, Kottiri BJ, Neaigus A, Curtis R, Vermund SH, Des Jarlais DC: Network-related mechanisms may help explain long-term HIV-1 seroprevalence levels that remain high but do not approach population-group saturation. Am J Epidemiol 2000, 152(10):913-922.

3. Nemoto T, Operario D, Keatley J, Han L, Soma T: HIV risk behaviors among male-to-female transgender persons of color in San Francisco. Am J Public Health 2004, 94(7):1193-1199.

4. Wilson EC, Garofalo R, Harris DR, Belzer M: Sexual risk taking among transgender male-to-female youths with different partner types. Am J Public Health 2010, 100(8):1500-1505.

5. Operario D, Nemoto T: HIV in transgender communities: syndemic dynamics and a need for multicomponent interventions. J Acquir Immune Defic Syndr 2010, 55(Suppl 2):S91-S93.

6. Nemoto T, Bodeker B, Iwamoto M, Sakata M: Practices of receptive and insertive anal sex among transgender women in relation to partner types, sociocultural factors, and background variables. AIDS Care 2014, 26(4):434-440.

7. Bowers JR, Branson CM, Fletcher J, Reback CJ: Differences in substance use and sexual partnering between men who have sex with men, men who have sex with men and women and transgender women. Cult Health Sex 2011, 13(6):629-642.

8. Reback CJ, Fletcher JB: HIV prevalence, substance use, and sexual risk behaviors among transgender women recruited through outreach. AIDS Behav 2014, 18(7):1359-1367.

9. Santos GM, Rapues J, Wilson EC, Macias O, Packer T, Colfax G, Raymond HF: Alcohol and substance use among transgender women in San Francisco: prevalence and association with human immunodeficiency virus infection. Drug Alcohol Rev 2014, 33(3):287-295.

10. Kammerer N, Mason T, Connors M: Transgender Health and Social Service needs in the context of HIV Risk. In Transgender and HIV: Risks, Prevention and Care. Edited by Bockting W, Kirk S. Binghamton, NY: Haworth Press; 2001.

11. Sevelius JM, Reznick OG, Hart SL, Schwarcz S: Informing interventions: the importance of contextual factors in the prediction of sexual risk behaviors among transgender women. AIDS Educ Prev 2009, 21(2):113-127.

12. Wallace PM: Finding Self: A qualitative study of transgender, transitioning, and adulterated silicone. Health Educ J 2010, 69(4):439-446.

13. Wilson E, Rapues J, Jin H, Raymond HF: The use and correlates of illicit silicone or "Fillers" in a population-based sample of transwomen, San Francisco, 2013. J Sex Med 2014, 11(7):1717-1724.
14. Hart TA, Wolitski RJ, Purcell DW, Purcell DW, Parsons JT, Gomez CA, Seropositive Urban Men's Study Team : Partner awareness of the serostatus of HIV-seropositive men who have sex with men: impact on unprotected sexual behavior. AIDS Behav 2005, 9(2):155-166.

15. Golden MR, Dombrowski JC, Kerani RP, Steckler JD: Failure of serosorting to protect African American men who have sex with men from HIV infection. Sex Transm Dis 2012, 39(9):659-664.

16. Strathdee SA, Sherman SG: The role of sexual transmission of HIV infection among injection and non-injection drug users. J Urban Health 2003, 80(4 Suppl 3):iii7-iii14.

17. Bohl DD, Raymond HF, Arnold M, McFarland W: Concurrent sexual partnerships and racial disparities in HIV infection among men who have sex with men. Sex Transm Infect 2009, 85(5):367-369.

18. Rapues J, Wilson EC, Packer T, Colfax G, Raymond HF: Correlates of HIV infection among transfemales, San Francisco, 2010: results from a respondent-driven sampling study. Am J Public Health 2013, 103(8):1485-1492.

19. Doherty IA, Padian NS, Marlow C, Aral SO: Determinants and consequences of sexual networks as they affect the spread of sexually transmitted infections. J Infect Dis 2005, 191(Suppl 1):S42-S54.

20. Pinkerton SD, Galletly CL, McAuliffe TL, DiFranceisco W, Raymond HF, Chesson HW: Aggregate versus individual-level sexual behavior assessment: how much detail is needed to accurately estimate HIV/STI risk? Eval Rev 2010, 34(1):19-34.

21. Ma X, Zhang Q, He X, Sun W, Yue H, Chen S, Raymond HF, Li Y, Xu M, Du H, McFarland W: Trends in prevalence of HIV, syphilis, hepatitis $C$, hepatitis $B$, and sexual risk behavior among men who have sex with men. Results of 3 consecutive respondent-driven sampling surveys in Beijing, 2004 through 2006. J Acquir Immune Defic Syndr 2007, 45(5):581-587.

22. McFarland W, Chen YH, Nguyen B, Colfax G, Mehrtens J, Robertson T, Stall R, Levine D, Truong HM: Behavior, intention or chance? A longitudinal study of HIV seroadaptive behaviors, abstinence and condom use. AIDS Behav 2012, 16(1):121-131.

23. McFarland W, Chen YH, Raymond HF, Nguyen B, Colfax G, Mehrtens J, Robertson T, Stall R, Levine D, Truong HM: HIV seroadaptation among individuals, within sexual dyads, and by sexual episodes, men who have sex with men, San Francisco, 2008. AIDS Care 2011, 23(3):261-268.

24. Chen YH, Vallabhaneni S, Raymond HF, McFarland W: Predictors of serosorting and intention to serosort among men who have sex with men, San Francisco. AIDS Educ Prev 2012, 24(6):564-573.

25. Bohl DD, McFarland W, Raymond HF: Improved measures of racial mixing among men who have sex with men using Newman's assortativity coefficient. Sex Transm Infect 2011, 87(7):616-620.

26. Sudhinaraset M, Raymond HF, McFarland W: Convergence of HIV prevalence and inter-racial sexual mixing among men who have sex with men, San Francisco, 2004-2011. AIDS Behav 2013, 17(4):1550-1556.

27. Doherty IA, Schoenbach VJ, Adimora AA: Sexual mixing patterns and heterosexual HIV transmission among African Americans in the southeastern United States. J Acquir Immune Defic Syndr 2009, 52(1):114-120.

28. Choi KH, Han CS, Hudes ES, Kegeles S: Unprotected sex and associated risk factors among young Asian and Pacific Islander men who have sex with men. AIDS Educ Prev 2002, 14(6):472-481.

29. Bingham TA, Harawa NT, Johnson DF, Nguyen B, Colfax G, Mehrtens J, Robertson T, Stall R, Levine D, Truong HM, Secura G, Mackellar DA, Valleroy $L$ : The effect of partner characteristics on HIV infection among African American men who have sex with men in the Young Men's Survey, Los Angeles, 1999-2000. AIDS Educ Prev 2003, 15(1 Suppl A):39-52.

30. Newcomb ME: Moderating effect of age on the association between alcohol use and sexual risk in MSM: evidence for elevated risk among younger MSM. AIDS Behav 2013, 17(5):1746-1754.

31. Malloy TE, Fisher WA, Albright L, Misovich S, Fisher JD: Interpersonal perception of the AIDS risk potential of persons of the opposite sex. Health Psychol 1997, 16(5):480-486.

32. Operario D, Nemoto T, Iwamoto M, Moore T: Unprotected sexual behavior and HIV risk in the context of primary partnerships for transgender women. AIDS Behav 2011, 15(3):674-682.

33. Nagaraj S, Segura ER, Peinado J, Konda KA, Segura P, Casapia M, Ortiz A, Montano SM, Clark JL, Sanchez J, Lama JR, Peruvian HIV Sentinel Surveillance Working Group: A cross-sectional study of knowledge of sex partner serostatus among high-risk Peruvian men who have sex with 
men and transgender women: implications for HIV prevention. BMC Public Health 2013, 13:181.

34. Vallabhaneni $S, L i X$, Vittinghoff E, Donnell D, Pilcher CD, Buchbinder SP: Seroadaptive practices: association with HIV acquisition among HIV-negative men who have sex with men. PLoS One 2012, 7(10):e45718.

35. McConnell JJ, Bragg L, Shiboski S, Grant RM: Sexual seroadaptation: lessons for prevention and sex research from a cohort of HIV-positive men who have sex with men. PLoS One 2010, 5(1):e8831.

36. Millett GA, Peterson JL, Wolitski RJ, Stall R: Greater risk for HIV infection of black men who have sex with men: a critical literature review. Am J Public Health 2006, 96(6):1007-1019.

37. Operario D, Nemoto T, Iwamoto M, Moore T: Risk for HIV and unprotected sexual behavior in male primary partners of transgender women. Arch Sex Behav 2011, 40(6):1255-1261.

38. Santis J: HIV infection risk factors among male-to-female transgender persons: a review of the literature. J Assoc Nurses AIDS Care 2009, 20(5):362-372.

doi:10.1186/1471-2334-14-430

Cite this article as: Wilson et al.: Sexual mixing and the risk environment of sexually active transgender women: data from a respondent-driven sampling study of HIV risk among transwomen in San Francisco, 2010. BMC Infectious Diseases 2014 14:430.

\section{Submit your next manuscript to BioMed Central and take full advantage of:}

- Convenient online submission

- Thorough peer review

- No space constraints or color figure charges

- Immediate publication on acceptance

- Inclusion in PubMed, CAS, Scopus and Google Scholar

- Research which is freely available for redistribution 